\title{
Pigmented and Depressed Facial Scar after an External Injury: Successful Treatment with 1,064-nm Picosecond Neodymium: Yttrium-Aluminum-Garnet Laser Simply Combined with a Fractional Beam-Splitter Handpiece
}

\author{
Won Soon Chung ${ }^{1}$ \\ Hannah Lee ${ }^{2}$ \\ Tae-Gyun $\mathrm{Kim}^{3}$
}

${ }^{1}$ Yonsei Star Skin \& Laser Clinic, Seoul, Korea ${ }^{2}$ Lexington Christian Academy, Lexington, MA, USA

${ }^{3}$ Department of Dermatology, Severance Hospital, Cutaneous Biology Research Institute, Yonsei University College of Medicine, Seoul, Korea
Received June 8, 2018

Accepted June 13, 2018

\section{Correspondence}

Tae-Gyun Kim

Department of Dermatology, Severance Hospital, Cutaneous Biology Research Institute, Yonsei University College of Medicine, 50-1 Yonsei-ro, Seodaemun-gu, Seoul 03722, Korea

Tel.: +82-2-2228-2080

Fax: +82-2-393-9157

E-mail: tgmed83dyuhs.ac

(C) Korean Society for Laser Medicine and Surgery

(c) This is an open access article distributed under the terms of the Creative Commons Attribution NonCommercial License (http://creativecommons.org/ licenses/by-nc/4.0) which permits unrestricted noncommercial use, distribution, and reproduction in any medium, provided the original work is properly cited.
A 29-year-old man presented with facial scars one and half years after an external injury. A physical examination revealed a slightly depressed linear scar with bluish brown pigments from traumatic tattoos on the nasal root. He was treated with a 1,064-nm picosecond neodymium:yttriumaluminum-garnet (Nd:YAG) laser combined with a fractional picosecond mode by integrating a holographic beam splitter handpiece. After five sessions of this simple combined treatment modality, the patient achieved significant clinical improvement with no downtime period. To the best of the authors' knowledge, this is the first case of the effective treatment of facial scars after a skin injury using a 1,064-nm picosecond $\mathrm{Nd}$ :YAG laser together with a simple combination of a fractional beam splitter handpiece, successfully targeting both the traumatic tattoos and depressed scars.

\section{Key words}

Beam-splitter handpiece; Depressed scar; Picosecond laser; Tattoo 


\section{INTRODUCTION}

Skin scarring is a consequence of skin wound healing process influenced by multiple genetic and environmental factors. ${ }^{1}$ There are several clinical categories of skin scarring such as, depressed scars, hypertrophic scars, and keloid scars. ${ }^{2}$ According to the etiology of skin scarring, scars resulting from external injuries frequently present more complicated features including hypertrophy, depression, erythema, hyperpigmentation, and traumatic tattoos. Therefore, those scars tend to be challenging to treat due to their heterogeneous nature of shape, depth, and colors, which raise the needs for combination laser therapy to achieve optimal therapeutic outcomes. Here, we report a case of pigmented and depressed scars successfully treated with a 1,064-nm picosecond neodymium:yttrium-aluminum-garnet (Nd:YAG) laser simply combined with an additional fractional beam splitter handpiece for targeting both tattoos and depression.

\section{CASE REPORT}

A 29-year-old Korean male presented to our clinic with a linear facial scar following a traumatic external injury one and half year prior to his visit. Physical examination revealed a linearly depressed scar with bluish brown pigments on the nasal root (Fig. 1A). After initial evaluation, we performed total five sessions of laser therapy at one month interval using the 1,064-nm picosecond Nd:YAG laser (PicoWay ${ }^{\circledR}$; Syneron-Candela Corporation, Wayland, MA, USA) with the fluence of range between 1.4-2.4 J/ $\mathrm{cm}^{2}$ for treating traumatic tattoos of the scar. From the second to the final sessions of the treatment, the patient was additionally subjected to the 1,064-nm picosecond $\mathrm{Nd}$ :YAG laser therapy equipped with a holographic beamsplitting fractional optic handpiece (PicoWay Resolve ${ }^{\circledR}$; Syneron-Candela Corporation) with the fluence of range between 1.5-1.7 J/ $\mathrm{cm}^{2}$ for total three passes per each treatment session for treating depression of the scar. Before treatment, an explanation of the risks, benefits, and potential complications of the procedure were given, and written informed consent was obtained. Anesthesia was achieved with a topical EMLA cream (AstraZeneca $A B$, Södertälje, Sweden) for 1 hour before each treatment. After two treatments in 2 months (two picosecond laser and one picosecond laser armed with beam splitting handpiece), there was a significant improvement in the pigmentation due to traumatic tattoos and the appearance of depression (Fig. 1B). A total of five sessions of laser therapy resulted in an outstanding clinical outcome without any delayed down-time and inconvenient side effects (Fig. 1C).

\section{DISCUSSION}

Traumatic external injuries can cause a highly heterogeneous features of scar phenotypes as a combination of erythema, pigment, hypertrophy, and atrophy. Especially, skin scarring of this etiology frequently leaves traumatic tattoos within the intradermal area of scar lesions which result in deleterious cosmetic issues. ${ }^{3}$ Those characteristics have led to test a wide range of laser modalities to achieve the optimal treatment outcome against the post-traumatic pigmented scars. It has been well established that traumatic tattoos would be efficiently treated by 1,064-nm Q-switched Nd:YAG laser with nanosecond pulse duration. ${ }^{4}$ However, based on the theory of selective photothermolysis, ${ }^{5}$ lasers emitting nanosecond pulse durations are not shorter enough to attain the most efficient tattoo removal. In addition, Asian patients treated with those nanosecond lasers are prone to be complicated by post-laser hyperpigmentation and scarring due to higher epidermal melanin contents compared to those of Caucasians. $^{6.7}$ In light of this, almost twenty years ago Ross
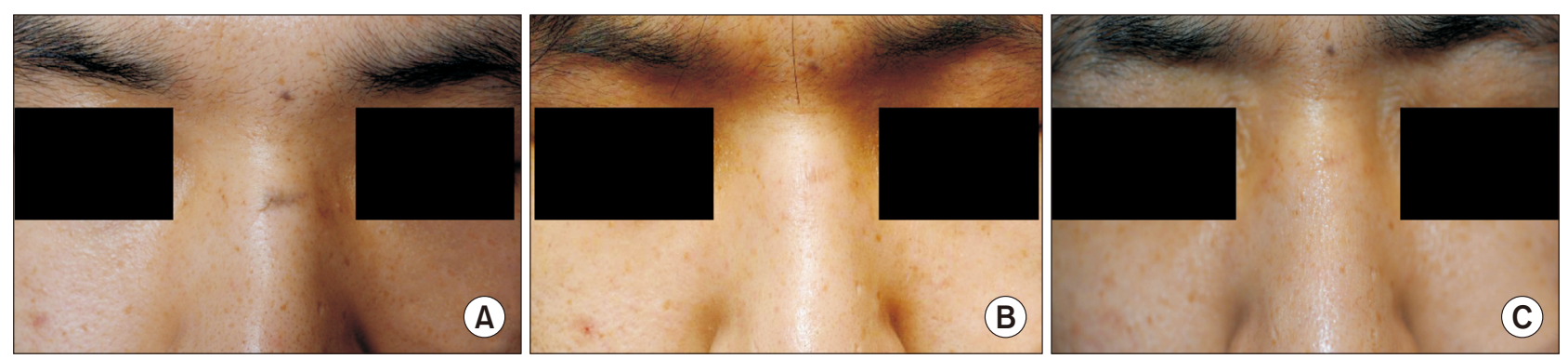

Fig. 1. Clinical improvement of the facial scar on the nasal root after treatment with five sessions of 1,064-nm picosecond neodymium:yttriumaluminum-garnet (Nd:YAG) laser simply combined with an additional holographic beam-splitter handpiece: (A) before the treatment, (B) after two sessions with laser treatment, and (C) before final session of the treatment. 
et al. first reported that Nd:YAG laser with picosecond pulses showed more superior therapeutic effect than nanosecond pulses in clearing black tattoos. ${ }^{8}$ In addition, it has been also demonstrated that picosecond-domain $\mathrm{Nd}$ :YAG laser therapy was highly efficacious in the treatment of traumatic tattoos in Asians with great safety profiles. ${ }^{9}$ In our case, we successfully treated hyperpigmented traumatic tattoos by applying 1,064-nm picosecond Nd:YAG laser. For covering linearly depressed area, we treated the patient by simply incorporating a novel holographic beam-splitter to achieve fractional picosecond laser beams. It has been recently described that laserinduced optical breakdown in the skin in conjunction with picosecond-domain pulse durations led to a significant improvement in scar treatment. ${ }^{10,11}$ Although the traditional non-ablative and ablative fractionated laser therapies are widely accepted to treat various types of scars, including the depressed one by targeting water absorption, those lasers are often accompanied by significant hyperpigmentation when treating more darkly pigmented skin, such as Asian skins. ${ }^{12}$ However, as in our patient, the subjects treated by split laser beams of picosecond laser usually experienced minimal or no downtime with an excellent clinical outcomes. ${ }^{13}$ Thus, we propose that 1,064$\mathrm{nm}$ picosecond-domain $\mathrm{Nd}$ :YAG laser combined with an additional holographic beam-splitter would be a highly effective and reliable therapeutic approaches for the mixed features of pigmented and depressed post-traumatic scars especially complicated by traumatic tattooing.

\section{REFERENCES}

1. Profyris C, Tziotzios C, Do Vale I. Cutaneous scarring: pathophysiology, molecular mechanisms, and scar reduction therapeutics Part I. The molecular basis of scar formation. J Am Acad Dermatol 2012;66:1-10.

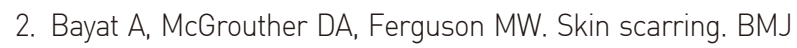

\section{3;326:88-92.}

3. Agris J. Traumatic tattooing. J Trauma 1976;16:798-802.

4. Suzuki $H$. Treatment of traumatic tattoos with the $Q$-switched neodymium:YAG laser. Arch Dermatol 1996;132:1226-9.

5. Anderson RR, Parrish JA. Selective photothermolysis: precise microsurgery by selective absorption of pulsed radiation. Science 1983;220:524-7.

6. Ho SG, Goh CL. Laser tattoo removal: a clinical update. J Cutan Aesthet Surg 2015;8:9-15.

7. Khunger N, Molpariya A, Khunger A. Complications of tattoos and tattoo removal: stop and think before you ink. J Cutan Aesthet Surg 2015;8:30-6.

8. Ross V, Naseef G, Lin G, Kelly M, Michaud N, Flotte TJ, et al. Comparison of responses of tattoos to picosecond and nanosecond Q-switched neodymium: YAG lasers. Arch Dermatol 1998;134:167-71.

9. Goo BL, Cho SB. Efficacy and safety of picosecond-domain neodymium-doped Yttrium aluminum garnet laser treatment on various causes of traumatic tattoos. Med Lasers 2016;5:905.

10. Habbema L, Verhagen R, Van Hal R, Liu Y, Varghese B. Efficacy of minimally invasive nonthermal laser-induced optical breakdown technology for skin rejuvenation. Lasers Med Sci 2013;28:935-40.

11. Brauer JA, Kazlouskaya V, Alabdulrazzaq H, Bae YS, Bernstein $\mathrm{LJ}$, Anolik R, et al. Use of a picosecond pulse duration laser with specialized optic for treatment of facial acne scarring. JAMA Dermatol 2015;151:278-84.

12. Mahmoud BH, Srivastava D, Janiga JJ, Yang JJ, Lim HW, Ozog DM. Safety and efficacy of erbium-doped yttrium aluminum garnet fractionated laser for treatment of acne scars in type IV to VI skin. Dermatol Surg 2010;36:602-9.

13. Bernstein EF, Schomacker KT, Basilavecchio LD, Plugis JM, Bhawalkar JD. Treatment of acne scarring with a novel fractionated, dual-wavelength, picosecond-domain laser incorporating a novel holographic beam-splitter. Lasers Surg Med 2017;49:796-802. 